
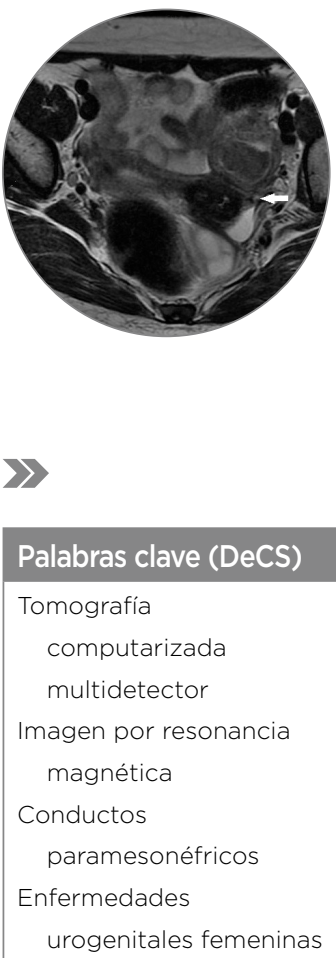

\begin{tabular}{|l|} 
Key words (MeSH) \\
Multidetector computed \\
tomography \\
Magnetic resonance \\
imaging \\
Mullerian ducts \\
Female urogenital \\
diseases \\
\hline
\end{tabular}

Médica, Universidad Pedagógica y Tecnológica de Colombia. Tunja, Colombia.

${ }^{2}$ Radiólogo, Universidad de Buenos Aires. Buenos Aires, Argentina.

Departamento de Radiología, Hospital Regional Manuela Beltrán. Socorro, Colombia.

\title{
Síndrome de Herlyn Werner Wünderlich. Presentación de caso y revisión de literatura
}

\author{
Herlyn Werner Wünderlich Syndrome. Presentation of Case and \\ Review of Literature
}

Maricela Fuentes Lizarazo

Crhistian de Arco Espinosa ${ }^{2}$

\begin{abstract}
Resumen
El síndrome de Herlyn-Werner-Wünderlich es una malformación mülleriana rara y poco diagnosticada, que se caracteriza por la triada de hemivagina obstruida, agenesia renal ipsilateral y útero didelfo; su diagnóstico suele ser tardío debido a la sintomatología inespecífica que produce: dolor abdominal, dismenorrea y masa abdominal palpable secundaria a hematocolpos. Esta entidad ocasiona graves alteraciones en la fertilidad. El uso de imágenes diagnósticas, como ecografía, tomografía y resonancia magnética, es imprescindible para diagnosticar esta anomalía. Se presenta el caso de una paciente de 12 años con esta condición y una revisión de literatura.
\end{abstract}

\section{Summary}

Herlyn Werner Wünderlich Syndrome is a rare and undiagnosed mullerian malformation, characterized by the triad of clogged hemivagina, ipsilateral renal agenesis and didelphic uterus; its diagnosis is usually late due to the unspecific symptomatology it produces: abdominal pain, dysmenorrhea and palpable abdominal mass secondary to hematocolpos, causing serious changes in fertility. The use of diagnostic images such as ultrasound, tomography and magnetic resonance imaging is essential to diagnose this type of anomaly. The case of a 12-year-old patient with this condition and a review of the literature are presented.

\section{Introducción}

El síndrome de Herlyn-Werner-Wünderlich es un tipo de malformación mülleriana, rara, caracterizada por la triada de hemivagina obstruida, agenesia renal ipsilateral y útero didelfo, descrita por primera vez por Purslow en 1922 (1). Las malformaciones müllerianas son un grupo de entidades raras y muy poco diagnosticadas que causan sintomatología inespecífica en adolescentes que de no ser detectadas, pueden ocasionar alteraciones en la fertilidad y resultados obstétricos adversos (2).

La incidencia de este síndrome se desconoce debido a que gran parte de las pacientes cursan asintomáticas; sin embargo, se estima que para las anomalías müllerianas en general se encuentra en un 2-3 \% y el síndrome de Herlyn-Werner-Wünderlich representa un 0,1-3,5\% de estas anormalidades (3). En la actualidad, se han informado cerca de 200 casos. De manera individual también hay variación en la incidencia de cada uno de los componentes de la triada clásica; en el caso del útero didelfo es aproximadamente $1 / 2.000$ a 1/28.000; la incidencia de agenesia renal unilateral es $1 / 1.100$ y solo ocurre en el $43 \%$ de los casos y un $25-50 \%$ de las mujeres afectadas por el síndrome tiene anormalidades genitales asociadas (4).

Suele aparecer en adolescentes, pero gran parte de ellas puede tener menstruaciones normales (5), por lo que en la mayoría de los casos hay un retraso en el diagnóstico y tratamiento (6); sin embargo, aunque la mayoría de síntomas son inespecíficos, este síndrome se relaciona con dolor pélvico, que se inicia característicamente luego de la menarquia y es secundario a hematocolpos (7), dismenorrea recurrente e intensa, masa abdominal palpable asociada a hematocolpos o hematometra; de manera excepcional, se relaciona con infertilidad, fiebre o retención urinaria aguda (8). Además, es realmente difícil llegar a un diagnóstico preciso, ya que usualmente las pacientes ante la dismenorrea y el dolor abdominal suelen consumir analgésicosantinflamatorios y anticonceptivos orales, lo cual reduce aún más las posibilidades diagnósticas (9).

\section{Presentación del caso}

Paciente femenina de 12 años de edad, sin antecedentes patológicos, con dolor abdominal de larga data asociado a sangrado menstrual, consulta al servicio de urgencias por cuadro clínico de 3 días de evolución consistente en dolor abdominal de predominio en el mesogastrio, asociado a estreñimiento y emesis; refiere estar menstruando, niega otros síntomas asociados, al examen físico se encuentra dolor a la palpación en marco cólico, defensa a la palpación superficial sin signos de irritación peritoneal. Se realiza hemograma, uroanálisis 
y ecografía abdominal en la que se evidencia agenesia renal izquierda y hallazgos compatibles con hematocolpos, por lo que se le practica urografia por tomografía multicorte sin medio de contraste endovenoso (UROTAC) en la que se observa riñón derecho único (flecha blanca) e imagen quística al lado derecho del útero (flecha negra) (figura 1).

Se inicia manejo farmacológico, con el fin de disminuir la sintomatología producto del hematocolpos; se le practica tomografía axial computarizada (TAC) de abdomen y pelvis, en la que se observa riñón derecho único (flecha blanca) (figura 2) sin hallazgos concluyentes en cortes coronales por lo que no se realiza reporte de los mismos, y se consideró la necesidad de valoración por parte de ginecología pediátrica; sin embargo, como en la institución donde se atendía el caso no se contaba con dicha especialidad, se continuó manejo farmacológico para disminuir la sintomatología producto del hematocolpos.

A los 17 meses ingresó de nuevo la paciente por consulta externa de ginecología, refirió disminución de la sintomatología abdominopélvica posterior a manejo farmacológico; trae resultado de RM de abdomen y pelvis en la que se observa ausencia de riñón izquierdo, dos ovarios de forma y contornos normales, con múltiples imágenes quísticas de alta señal en secuencias con información T2, de diámetro variable entre 5 y 7 mm que ocupan el estroma ovárico (figura 3), útero bicorne bicoli con dos cavidades endometriales (flechas blancas) (figura 4). Con estos hallazgos en RM se diagnosticó el síndrome de Herlyn-Werner-Wünderlich y se continuó manejo farmacológico.

\section{Discusión}

Las anomalías características de este síndrome ocurren hacia la octava semana de gestación, debido a que no se desarrollan o se fusionan los conductos müllerianos (1), lo cual genera su triada característica: útero didelfo, septo vaginal obstructivo y agenesia renal homolateral. El septo vaginal es consecuencia de la falta de fusión lateral de los conductos müllerianos descendentes, lo cual origina dos hemivaginas. Esta malformación altera el drenaje normal del material hemático, lo que da lugar a hematocolpos. El útero didelfo ocurre como consecuencia de la falta de fusión de los conductos paramesonéfricos, y da como resultado dos cavidades uterinas cada una con una trompa. Por último, se encuentra la agenesia renal ipsilateral, que se debe a la alteración en el desarrollo de la porción caudal de uno de los conductos de Wolf (7).

Durante la niñez, las pacientes portadoras del síndrome cursan asintomáticas; sin embargo, durante la adolescencia se manifiesta de manera inespecífica. El principal síntoma es dolor pélvico cíclico que puede estar asociado al dolor menstrual normal; no obstante, este tipo de dolor suele ser recurrente y progresivo, secundario al hematocolpos (10). Gran número de pacientes son subdiagnosticadas por la ausencia de irregularidades en el ciclo menstrual, generalmente uno de los úteros no se encuentra obstruido y es funcional. Por otra parte, las pacientes con irregularidades menstruales a menudo son tratadas con anticonceptivos orales y antinflamatorios, con mejoría de la dismenorrea. Sin embargo, a largo plazo suelen desarrollar dolor abdominal secundario al hematocolpos (11). Las pacientes rara vez cursan con sintomatología como infertilidad, náuseas, fiebre (8), o presentarse retención urinaria y constipación 10-12 meses después de la menarquia (12). En algunos casos, el útero didelfo se asocia con alteraciones reproductivas abortos, partos pretérmino, disfunción placentaria (13), así como también con piosálpinx, piohematocolpos, pelviperitonitis, endometriosis y adherencias pélvicas (9).
Entre los diagnósticos diferenciales se deben tener en cuenta algunas anomalías del desarrollo uterino, como útero bicorne, útero unicorne con cuerno contralateral rudimentario no comunicante, himen imperforado e hipoplasia o agenesia de cuello uterino (7). El diagnóstico temprano permite prevenir complicaciones relacionadas con el flujo retrógrado y la consecuente endometriosis, y preservar la fertilidad (14).

Aunque este síndrome se descubre poco después de la menarquia, es conveniente sospechar alteraciones müllerianas en caso de encontrar hallazgos como riñón displásico multiquístico o agenesia renal en un feto (15).

La técnica de imágenes diagnósticas más utilizada en ginecología es la ecografía transvaginal. Es segura, de fácil acceso y bajo costo. Permite el diagnóstico diferencial de la patología anexial y endometrial (16). Su uso se debe considerar de primera línea en la mayoría de escenarios clínicos (17); sin embargo, surge la necesidad de complementarla con otros métodos de imagen en patologías complejas, como es el caso del síndrome de Herlyn-Werner-Wünderlich. La TAC suele utilizarse en casos de dolor abdominal bajo o pélvico agudo inespecífico, útil en el diagnóstico diferencial de urgencias ginecológicas. La RM es concluyente en la mayoría de los casos y en la actualidad puede considerarse como la prueba de elección en la diferenciación de las patologías ginecológicas congénitas, inflamatorias y tumorales (18), ya que permite la caracterización del contenido del canal endometrial y cervical, en caso de que se sospeche la presencia de útero septado. Su precisión en el diagnóstico de malformaciones uterinas ha sido bien establecida, y es en algunas series hasta del $100 \%$ (19). A pesar de la importancia tanto de la TAC como de la RM, su uso debe restringirse a condiciones específicas, en especial para caracterizar lesiones que no son completamente evaluables por ultrasonido. La laparoscopia puede ayudar a distinguir rápidamente entre un útero simple o uno duplicado; sin embargo, no se recomienda como método estándar de diagnóstico (12). Es útil para confirmar el diagnóstico cuando las imágenes no son concluyentes (20).

La mayoría de anomalías müllerianas no requieren tratamiento. En este síndrome el manejo consiste generalmente en la escisión de la hemivagina obstruida, con el fin de aliviar el dolor pélvico y los hematocolpos. El manejo quirúrgico se lleva a cabo en dos etapas, en la primera etapa la cirugía pretende drenar el hematocolpos y la siguiente etapa busca remodelar la vagina (11). En la actualidad se busca disminuir la necesidad de reintervenciones con el fin de minimizar el riesgo de obstrucciones postoperatorias y la necesidad de usar moldes vaginales y dilatadores (21). La histerectomía total o unilateral se puede considerar en casos en los que la resección del septo no es posible o en pacientes con estenosis recurrente e infección uterina grave (14). Las pacientes con útero didelfo tienen una alta probabilidad de tener un embarazo satisfactorio; no obstante, la tasa de abortos es alta, $74 \%$, de parto pretérmino en $22 \%$ y de requerimiento de cesárea en $82 \%$ (20). En caso de adolescentes se puede posponer la cirugía, la cual no se considera una opción inmediata y el tratamiento se puede llevar a cabo mediante análogos de la gonadotropina para mantener la amenorrea (14).

El pronóstico de estas pacientes es bueno, aunque la mayor preocupación es que se vea gravemente afectada la fertilidad; el $80 \%$ de las pacientes puede llegar a concebir, pero hay alta probabilidad de aborto y parto pretérmino. Por lo tanto, es de gran importancia realizar el diagnóstico temprano de estas pacientes, con el fin de evitar el riesgo de endometriosis e infertilidad (14). 


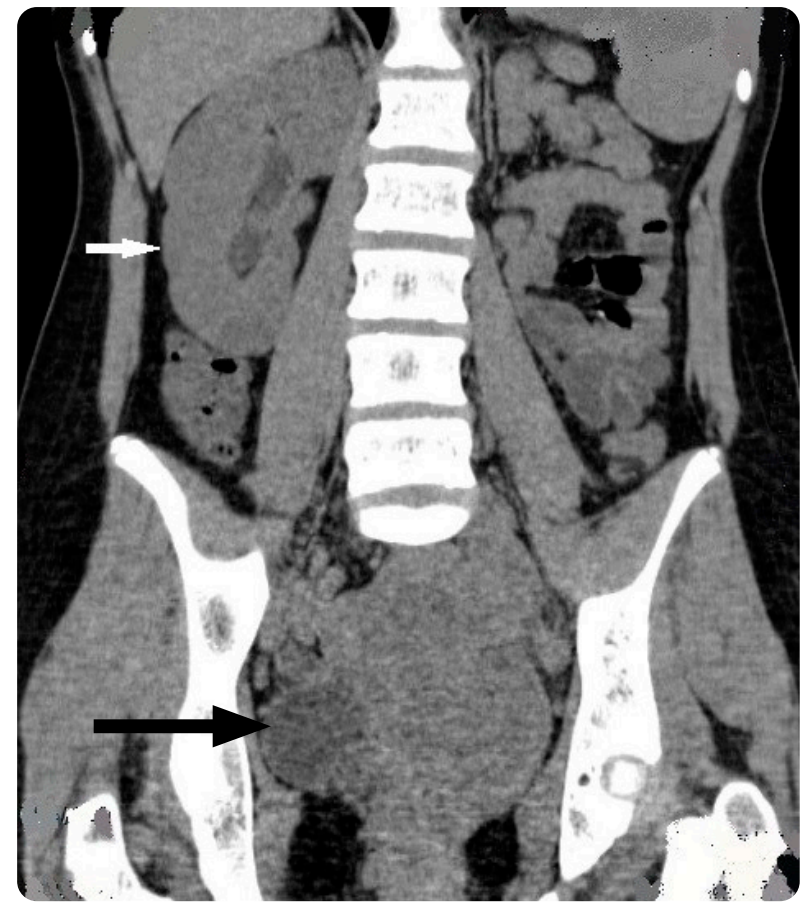

Figura 1. UROTAC, corte coronal en ventana de tejidos blandos: fosa renal izquierda vacía por agenesia congénita. Riñón derecho ligeramente aumentado de tamaño por hipertrofia compensatoria (flecha blanca), imagen quística del lado derecho del útero (flecha negra).

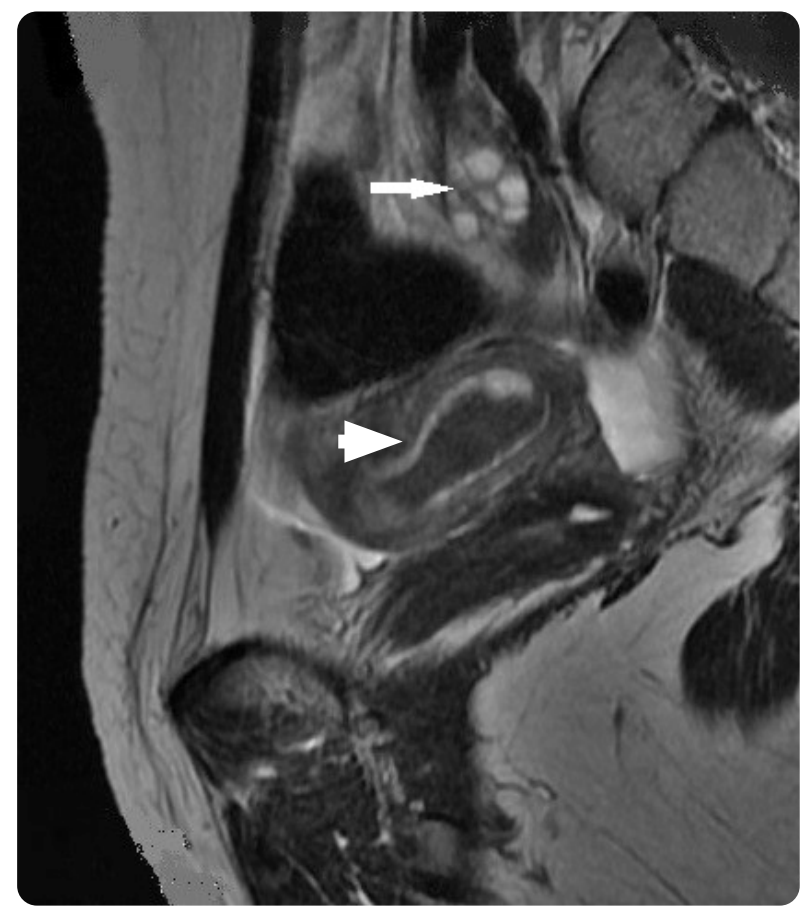

Figura 3. RM de abdomen y pelvis, corte sagital con imagen ponderada en T2: se observan dos cavidades uterinas, una de las cuales con intensidad de señal heterogénea en su interior por presencia contenido hemático (cabeza de flecha blanca); el cuello uterino y la vagina tienen apariencia normal. En la parte superior se identifica uno de los ovarios de morfología normal, con múltiples folículos antrales en su interior (flecha blanca).

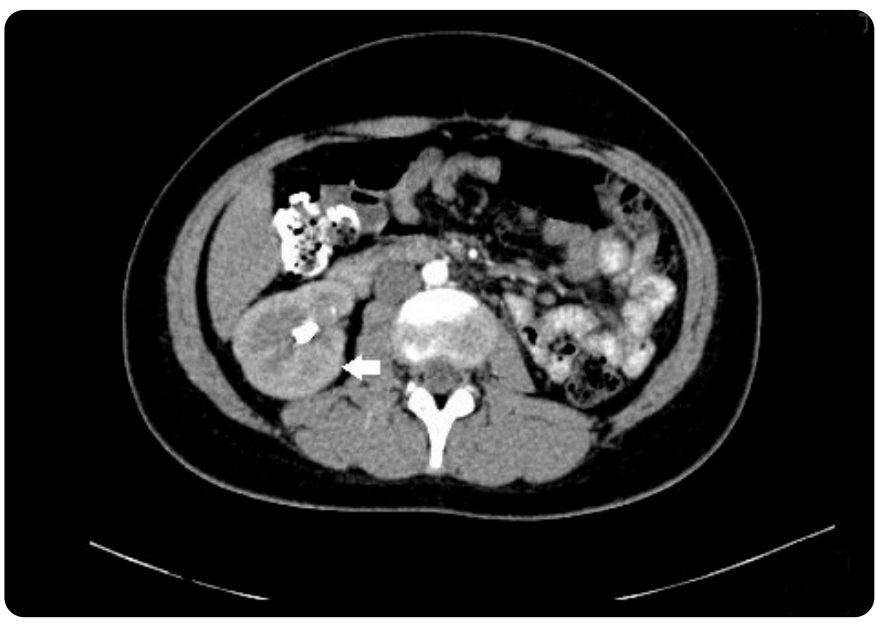

Figura 2. TAC de abdomen y pelvis con medio de contraste oral y endovenoso, corte axial: fosa renal izquierda vacía por agenesia congénita. Riñón derecho con realce normal, sin lesiones focales (flecha blanca).

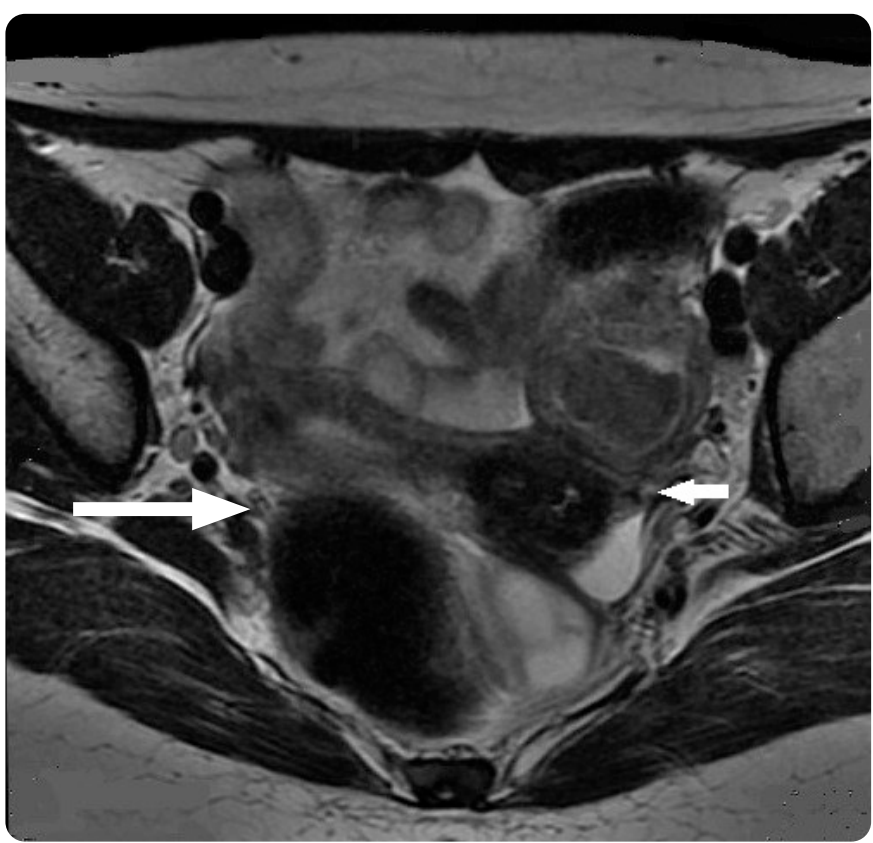

Figura 4. RM de abdomen y pelvis, corte axial con imagen ponderada en T2: se observan dos cavidades uterinas, una hacia la hemipelvis izquierda y otra hacia la hemipelvis derecha (flechas blancas) 


\section{Conclusiones}

En ginecología, el uso de imágenes diagnósticas como TAC y RM, métodos de extraordinaria resolución, se utilizan para casos complejos con el fin de resolver las dudas que surgen en la ecografía convencional. Sus índices diagnósticos son similares a la ecografía en muchas de las patologías benignas que generan dolor pélvico.

En el caso del síndrome de Herlyn-Werner-Wünderlich, anomalía compleja del sistema reproductor femenino, que generalmente se diagnostica tardíamente debido a su sintomatología inespecífica, el uso de imágenes diagnósticas permite hallar la triada que lo caracteriza. Se sospecha en una ecografía convencional, pero la caracterización definitiva debe ser mediante RM.

$\mathrm{Su}$ tratamiento inicial se enfoca en la disminución del dolor con suplementación hormonal y, posteriormente, en la resección del septo vaginal y el drenaje del hematocolpos con el fin minimizar los efectos adversos en la fertilidad de las pacientes, disminuyendo los abortos y los partos pretérmino.

Actualmente, la selección y uso correcto de los diferentes métodos de imágenes disponibles constituye uno de los pilares para el adecuado diagnóstico en ginecología; sin embargo, el uso de cada uno debe restringirse a condiciones específicas.

\section{Referencias}

1. Al-Jaroudi D, Khan F, Kaddour O, et al. Hysteroscopic resection of vaginal septum under ultrasound guidance in OHVIRA Syndrome with preservation of hymen. Int J Curr Advanc Res. 2015;4:450-3.

2. Afrashtehfar C, Piña-García A, Afrashtehfar K. Malformaciones müllerianas. Síndrome de hemivagina obstruida y anomalía renal ipsilateral (OHVIRA). Cir Cir 2014;82:46071. doi: https://www.redalyc.org/articulo.oa?id=66231427016

3. Lewis P, Zarariya A, Daver R, et al. An interesting case report on Herlyn-WernerWunderlich syndrome with hematocolpos and communicating blind duplex ureters. Int J Interdisc Multidisc Stud (IJIMS). 2015;2:163-7. doi: https://www.researchgate. net/publication/285220046

4. Berni R, Taboada D, Benítez Z. Síndrome de Herlyn Werner Wünderlich. Reporte de tres casos. Rev Salud Pública Parag. 2012;2:44-8.

5. Jaiprakash T, Saxena R, Pandey P. Obstructed hemivagina with ipsilateral renal anomaly (OHVIRA) syndrome - a rare congenital anomaly. J Genit Syst Disor. 2013;2:2-3. doi:10.4172/2325-9728.1000110

6. Patil B, Mysore R. Uterine didelphys with obstructed hemivagina and ipsilateral renal anomaly - OHVIRA syndrome: a rare congenital anomaly. Int J Reprod Contracept Obstet Gynecol. 2015;4:889-92. doi: http://dx.doi.org/10.18203/2320-1770. ijrcog20150121

7. Ahualli J, Méndez L, Ravera M, et al. Síndrome de Herlyn-Werner-Wünderlich: a propósito de un caso. RAR. 2011;75:203-6.

8. Ozturk H, Dagistan E, Ozlu T. Role of OHVIRA syndrome in renal agenesis: a case report. Ped Urol Case Rep. 2014;1:5-11. doi: http://dx.doi.org/10.14534/ PUCR.201424452

9. Del Vescovo R, Battisti S, Di Paola V, et al. Herlyn-Werner-Wunderlich syndrome: MRI findings, radiological guide (two cases and literature review), and differential diagnosis. BMC Medical Imaging. 2012;12:1-10. doi: 10.1186/1471-2342-12-4

10. Quintana R, Pinardo A, Calvo M, et al. OHVIRA Syndrome, knowledge and review. Eur Soc Radiol. 2015;1-19. doi: http://dx.doi.org/10.1594/ecr2015/C-1526

11. Da L, Chou L, Modh M. OHVIRA Syndrome obstructed hemivagina and ipsilateral renal anomaly and variants. Curr Women's Health Rev. 2014;10:22-5. doi: 10.2174/ 157340481001141030090716

12. Shah D, Laufer M. Obstructed hemivagina and ipsilateral renal anomaly (OHVIRA) syndrome with a single uterus. Fertility Sterility. 2011;96:39-41. https://doi org/10.1016/j.fertnstert.2011.05.013

13. Amin M. Uterus didelphys with obstructed hemivagina and ipsilateral renal anomaly (OHVIRA syndrome): A case report. J Ped Surg Case Reports. 2014;410-2. https:// doi.org/10.1016/j.epsc.2014.08.009

14. Salomao P, Doski J. Herlyn-Werner-Wunderlich syndrome: a case report. Rev Bras Ginecol Obstet. 2015;37:192-6. https://doi.org/10.1590/SO100-720320150005077

15. Guillan-Maquieira C, Sánchez J, Méndez J. Síndrome OHVIRA (hemivagina obstruida y anomalía renal ipsilateral) asociado a útero didelfo. Prog Obstet Ginecol. 2012;55:281-4. https://doi.org/10.1016/j.pog.2012.02.009
16. Huete A, Craig J, Vial C, et al. Rol de la imagenología en el proceso diagnóstico de la patología ginecológica benigna. Rev. chil. obstet. ginecol. 2016;81:63-85. http:// dx.doi.org/10.4067/S0717-75262016000100011

17. Benacerraf B, Abuhamad A, Bromley B, et al. Consider ultrasound first for imaging the female pelvis. Am J Obstet Gynecol. 2015;212:450-5. doi: https://doi.org/10.1016/j. ajog.2015.02.015

18. Frutos F, Hijona J, Espejo S, Torres J. Indicaciones de la resonancia magnética en el diagnóstico ginecológico. Progresos Obstet Ginecol. 2010;53:308-14. doi: 10.1016/j. pog.2009.12.010

19. Siu A, Vargas V, Murcia F, et al. Síndrome de OHVIRA: características clínicas y complicaciones, nuestra experiencia. Cir Pediatr. 2019;32:11-6.

20. Aveiro A, Miranda V, Cabral C, et al. Herlyn-Werner-Wunderlich syndrome: a rare cause of pelvic pain in adolescent girls. BMJ Case Reports. 2011;1-3. doi: 10.1136/ bcr.04.2011.4147.

21. Kapoor Y, Sherke A, Sheikh N. OHVIRA Syndrome - A case report. Int J Develop Res. 2014;4:1919-22

\section{Correspondencia}

Maricela Fuentes Lizarazo

Carrera 9 \# 11-26, local 202

San Gil, Santander, Colombia

mari_fuen@hotmail.com

Recibido para evaluación: 20 de mayo de 2020

Aceptado para publicación: 10 de enero de 2021 